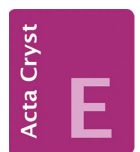

CRYSTALLOGRAPHIC COMMUNICATIONS

ISSN 2056-9890

Received 6 December 2016

Accepted 9 January 2017

Edited by T. J. Prior, University of Hull, England

Keywords: crystal structure; rubidium; peroxide; ammonia disolvate.

CCDC reference: 1526250

Supporting information: this article has supporting information at journals.iucr.org/e

\section{Crystal structure of rubidium peroxide ammonia disolvate}

\author{
Tobias GrassI and Nikolaus Korber*
}

Institut für Anorganische Chemie, Universität Regensburg, Universitätsstrasse 31, 93053 Regensburg, Germany. *Correspondence e-mail: nikolaus.korber@chemie.uni-regensburg.de

The title compound, $\mathrm{Rb}_{2} \mathrm{O}_{2} \cdot 2 \mathrm{NH}_{3}$, has been obtained as a reaction product of rubidium metal dissolved in liquid ammonia and glucuronic acid. As a result of the low-temperature crystallization, a disolvate was formed. To our knowledge, only one other solvate of an alkali metal peroxide is known: $\mathrm{Na}_{2} \mathrm{O}_{2} \cdot 8 \mathrm{H}_{2} \mathrm{O}$ has been reported by Grehl et al. [Acta Cryst. (1995), C51, 1038-1040]. We determined the peroxide bond length to be 1.530 (11) $\AA$, which is in accordance with the length reported by Bremm \& Jansen [Z. Anorg. Allg. Chem. (1992), 610, 64-66]. One of the ammonia solvate molecules is disordered relative to a mirror plane, with 0.5 occupancy for the corresponding nitrogen atom.

\section{Chemical context}

The crystal structure of the title compound was determined in the course of investigations regarding the reactivity of carbohydrates towards alkali metals and $\mathrm{NH}_{3}$ in solutions where liquid ammonia itself is used as solvent. The source of the peroxide anion could not be explicitly traced back but it seems to have its origin in oxygen gas from intruding atmosphere due to undetected leakage in the reaction vessel.

\section{Structural commentary}

The asymmetric unit contains one peroxide anion, two chargecompensating rubidium cations and two ammonia molecules (Fig. 1). Except for one nitrogen atom (N1, showing halfoccupancy) and one hydrogen atom $(\mathrm{H} 2 B)$, all other atoms are located on mirror planes. The anion is surrounded by four rubidium cations located around the girth of the peroxide ion (Fig. 2). This unit forms one-dimensional infinite strands by sharing one common edge of a distorted plane of four $\mathrm{Rb}$ ions
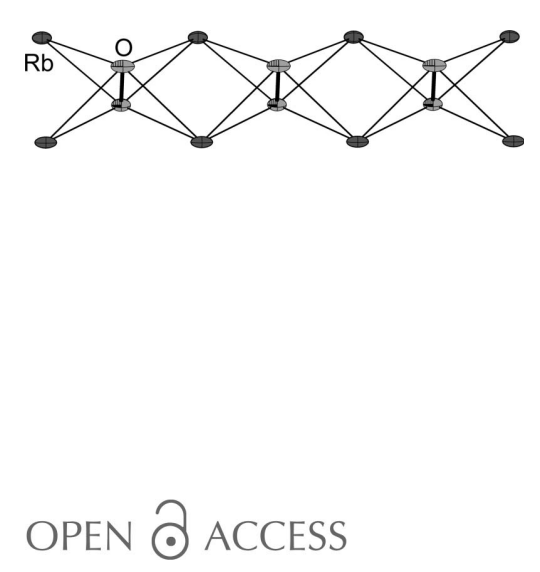

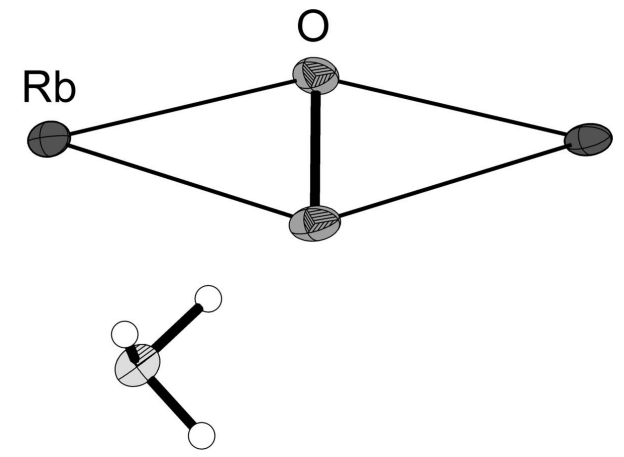

Figure 1

The asymmetric unit of the title compound, with the atom labeling and displacement ellipsoids drawn at the $50 \%$ probability level. 


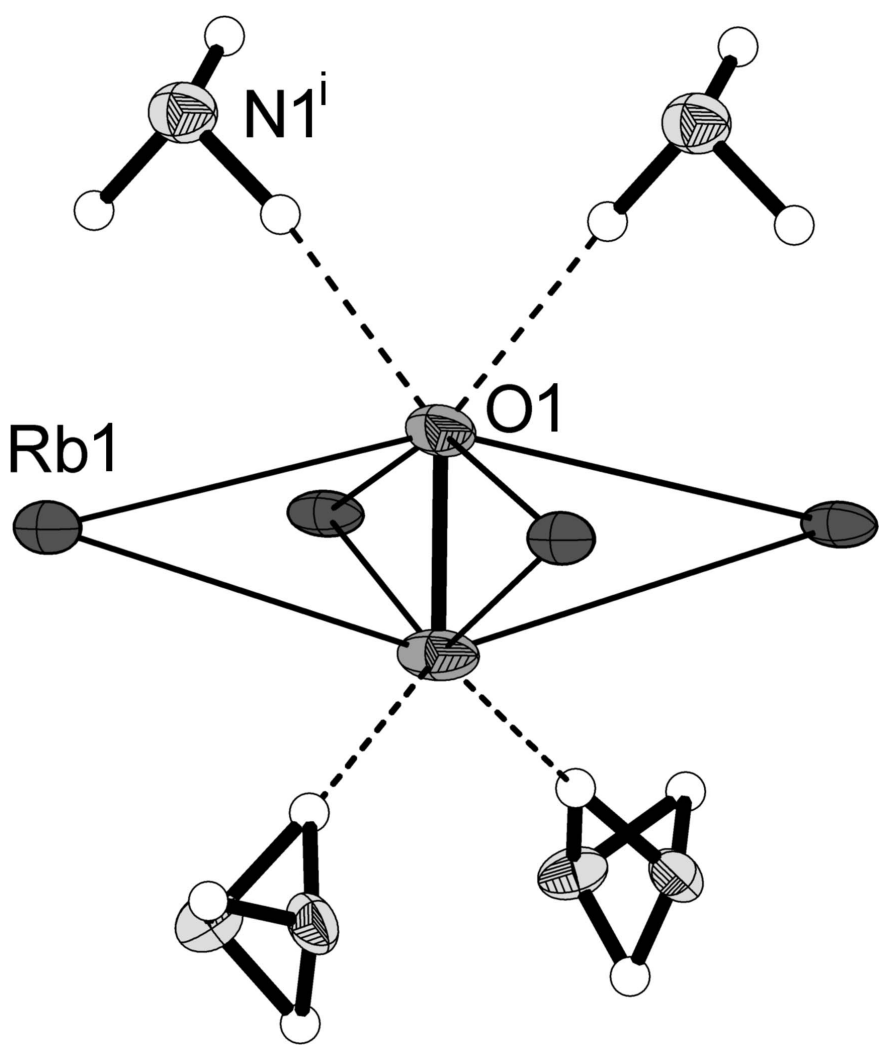

Figure 2

The environment of the peroxide anion. Displacement ellipsoids are drawn at the $50 \%$ probability level. [Symmetry code: (i) $-1+x, y, z$.]

(Fig. 3). This structural motif can also be observed in potassium acetylide $\mathrm{K}_{2} \mathrm{C}_{2}$ (Hamberger et al., 2012). The peroxide bond length was determined to be 1.530 (11) A. The anioncation contacts range between 2.790 (5) $\AA$ and 2.917 (6) $\AA$. The coordination number of the cations is 8 .

The $\mathrm{O}-\mathrm{O}$ bond length of the peroxide anion is longer than the value found in the literature based on the work of Föppl which is approximately $1.49 \AA$. In Fig. 4, a comparative view of bond lengths is presented based on the work of Bremm \& Jansen (1992), Föppl (1954, 1955, 1957) and Grehl et al. (1995).

\section{Supramolecular features}

Despite the low ammonia content, numerous hydrogen bonds can be observed and the $\mathrm{NH}_{3}$ molecules bridge the peroxide

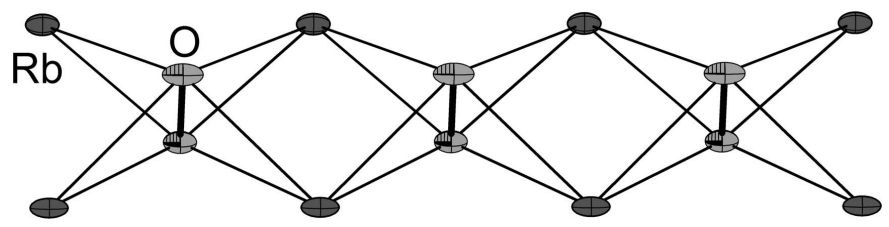

Figure 3

One-dimensional infinite strands formed by peroxide anions and rubidium cations. Displacement ellipsoids are drawn at the $50 \%$ probability level.
Table 1

Hydrogen-bond geometry $\left(\AA{ }^{\circ}\right)$.

\begin{tabular}{lllll}
\hline$D-\mathrm{H} \cdots A$ & $D-\mathrm{H}$ & $\mathrm{H} \cdots A$ & $D \cdots A$ & $D-\mathrm{H} \cdots A$ \\
\hline $\mathrm{N} 1-\mathrm{H} 1 A \cdots \mathrm{O} 2$ & $1.05(14)$ & $1.98(15)$ & $2.941(16)$ & $151(8)$ \\
$\mathrm{N} 1-\mathrm{H} 1 B \cdots \mathrm{O} 2^{\mathrm{i}}$ & $0.97(13)$ & $2.04(15)$ & $2.926(15)$ & $151(8)$ \\
$\mathrm{N} 1-\mathrm{H} 1 C \cdots 1^{\mathrm{ii}}$ & $0.82(14)$ & $3.07(16)$ & $3.597(16)$ & $125(11)$ \\
$\mathrm{N} 2-\mathrm{H} 2 A \cdots \mathrm{N} 2^{\mathrm{iii}}$ & $0.74(16)$ & $3.03(12)$ & $3.57(2)$ & $131(6)$ \\
$\mathrm{N} 2-\mathrm{H} 2 A \cdots \mathrm{N} 2^{\mathrm{iv}}$ & $0.74(16)$ & $3.03(12)$ & $3.57(2)$ & $131(6)$ \\
$\mathrm{N} 2-\mathrm{H} 2 A \cdots \mathrm{N} 2^{\mathrm{v}}$ & $0.74(16)$ & $3.03(12)$ & $3.57(2)$ & $131(6)$ \\
$\mathrm{N} 2-\mathrm{H} 2 B \cdots 1^{\mathrm{ii}}$ & $1.01(11)$ & $1.95(11)$ & $2.955(10)$ & $173(8)$ \\
\hline
\end{tabular}

Symmetry codes: (i) $x+\frac{1}{2},-y+\frac{3}{2},-z+\frac{1}{2}$; (ii) $x+1, y, z$; (iii) $-x+1,-y+1,-z+1$; (iv) $-x+1, y-\frac{1}{2},-z+1$; (v) $-x+1, y+\frac{1}{2},-z+1$.

anions. The peroxide anion shows five contacts to ammonia molecules, forming a three-dimensional network in the packing. The distances between donor and acceptor atoms ranges from 2.926(15) Angstrom to 3.597(16) Angstrom, which is commonly observed in ammoniates. Numerical details of the hydrogen-bonding interactions are given in Table 1.

\section{Synthesis and crystallization}

$500 \mathrm{mg}$ (2.58 mmol) D-glucuronic acid and $880 \mathrm{mg}$ $(10.29 \mathrm{mmol})$ rubidium were placed under an argon atmo-

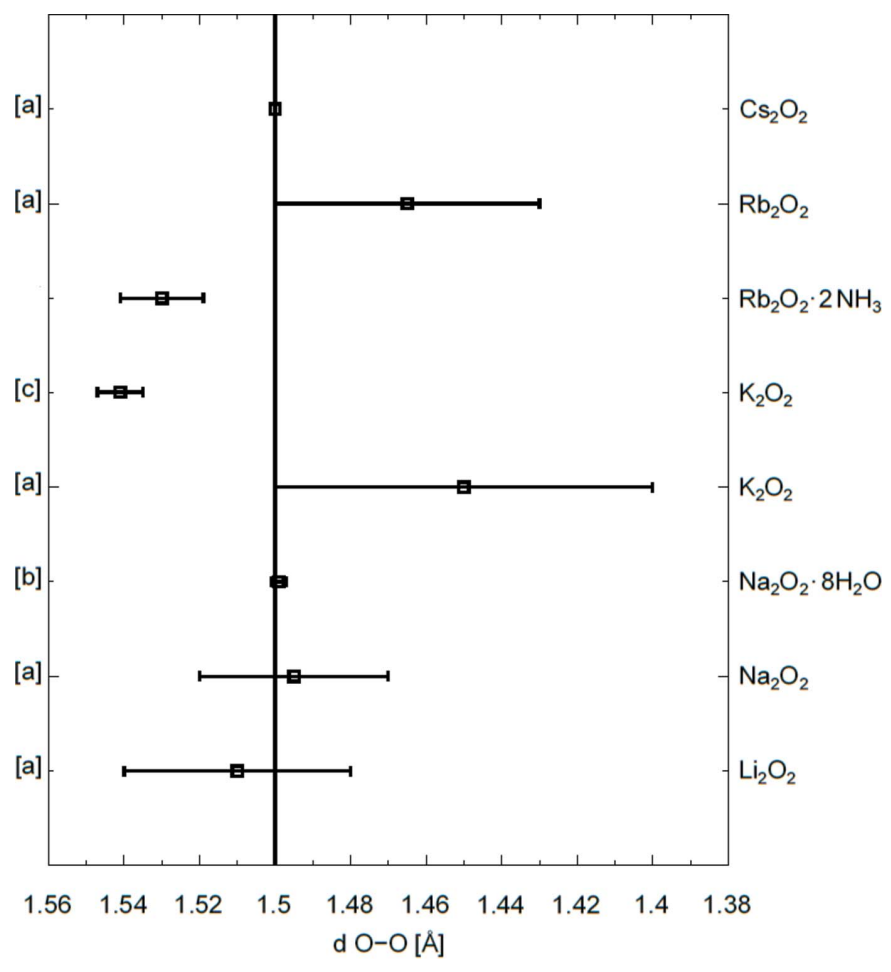
[a] Föppl $1954-1957$
[b] Grehl, Fröhlich \& Thiele 1995
[c] Bremm \& Jansen 1992

Figure 4

Comparison of peroxide bond lengths in different compounds. The vertical line shows the peroxide bond length commonly used in the literature. Each data point is shown with its standard uncertainties. 
Table 2

Experimental details.

\begin{tabular}{|c|c|}
\hline \multicolumn{2}{|l|}{ Crystal data } \\
\hline Chemical formula & $\mathrm{Rb}_{2} \mathrm{O}_{2} \cdot 2 \mathrm{NH}_{3}$ \\
\hline$M_{\mathrm{r}}$ & 237.01 \\
\hline Crystal system, space group & Orthorhombic, Pnma \\
\hline Temperature $(\mathrm{K})$ & 123 \\
\hline$a, b, c(\AA)$ & $7.3957(7), 4.0932(6), 18.1873(17)$ \\
\hline$V\left(\AA^{3}\right)$ & $550.57(11)$ \\
\hline$Z$ & 4 \\
\hline Radiation type & Mo $K \alpha$ \\
\hline$\mu\left(\mathrm{mm}^{-1}\right)$ & 17.66 \\
\hline Crystal size $(\mathrm{mm})$ & $0.24 \times 0.09 \times 0.08$ \\
\hline \multicolumn{2}{|l|}{ Data collection } \\
\hline Diffractometer & $\begin{array}{l}\text { Agilent SuperNova Dual Source } \\
\text { diffractometer with an Eos } \\
\text { detector }\end{array}$ \\
\hline Absorption correction & $\begin{array}{l}\text { Analytical [CrysAlis PRO } \\
\text { (Agilent, 2012), based on } \\
\text { expressions derived by Clark \& } \\
\text { Reid (1995)] }\end{array}$ \\
\hline$T_{\min }, T_{\max }$ & $0.064,0.354$ \\
\hline $\begin{array}{l}\text { No. of measured, independent and } \\
\text { observed }[I>2 \sigma(I)] \text { reflections }\end{array}$ & $2921,641,570$ \\
\hline$R_{\text {int }}$ & 0.057 \\
\hline$(\sin \theta / \lambda)_{\max }\left(\AA^{-1}\right)$ & 0.625 \\
\hline \multicolumn{2}{|l|}{ Refinement } \\
\hline$R\left[F^{2}>2 \sigma\left(F^{2}\right)\right], w R\left(F^{2}\right), S$ & $0.050,0.118,1.35$ \\
\hline No. of reflections & 641 \\
\hline No. of parameters & 51 \\
\hline H-atom treatment & Only $\mathrm{H}$-atom coordinates refined \\
\hline$\Delta \rho_{\max }, \Delta \rho_{\min }\left(\mathrm{e} \AA^{-3}\right)$ & $1.12,-1.79$ \\
\hline
\end{tabular}

Computer programs: CrysAlis PRO (Agilent, 2012), olex2.solve (Bourhis et al., 2015), SHELXL2014 (Sheldrick, 2015), DIAMOND (Brandenburg \& Putz, 2012) and OLEX2 (Dolomanov et al., 2009).

sphere in a reaction vessel and $25 \mathrm{ml}$ of dry liquid ammonia were condensed. The mixture was stored at $237 \mathrm{~K}$ for five days.
The flask was then stored at $161 \mathrm{~K}$ for several months. After that period, clear needle-shaped colorless crystals of the title compound could be found at the wall of the flask.

\section{Refinement}

Crystal data, data collection and structure refinement details are summarized in Table 2. The nitrogen atom N1 is disordered with 0.5 as the site occupation factor. All hydrogen atoms could be located in difference map and and their positions were refined freely with a common $U_{\text {iso }}(\mathrm{H})$ parameter. The isotropic displacement parameters were fixed to 0.025 .

\section{References}

Agilent (2012). CrysAlis PRO. Agilent Technologies Ltd, Yarnton, England.

Bourhis, L. J., Dolomanov, O. V., Gildea, R. J., Howard, J. A. K. \& Puschmann, H. (2015). Acta Cryst. A71, 59-75.

Brandenburg, K. \& Putz, H. (2012). DIAMOND. Crystal Impact GbR, Bonn, Germany.

Bremm, Th. \& Jansen, M. (1992). Z. Anorg. Allg. Chem. 610, 64-66. Clark, R. C. \& Reid, J. S. (1995). Acta Cryst. A51, 887-897.

Dolomanov, O. V., Bourhis, L. J., Gildea, R. J., Howard, J. A. K. \& Puschmann, H. (2009). J. Appl. Cryst. 42, 339-341.

Föppl, H. (1954). Angew. Chem. 66, 335-335.

Föppl, H. (1955). Angew. Chem. 67, 712-712.

Föppl, H. (1957). Z. Anorg. Allg. Chem. 291, 12-49.

Grehl, M., Fröhlich, R. \& Thiele, S. (1995). Acta Cryst. C51, 10381040.

Hamberger, M., Liebig, S., Friedrich, U., Korber, N. \& Ruschewitz, U. (2012). Angew. Chem. 124, 13181-13185.

Sheldrick, G. M. (2015). Acta Cryst. C71, 3-8. 


\section{supporting information}

Acta Cryst. (2017). E73, 200-202 [https://doi.org/10.1107/S2056989017000354]

\section{Crystal structure of rubidium peroxide ammonia disolvate}

\section{Tobias Grassl and Nikolaus Korber}

\section{Computing details}

Data collection: CrysAlis PRO (Agilent, 2012); cell refinement: CrysAlis PRO (Agilent, 2012); data reduction: CrysAlis PRO (Agilent, 2012); program(s) used to solve structure: olex2.solve (Bourhis et al., 2015); program(s) used to refine structure: SHELXL2014 (Sheldrick, 2015); molecular graphics: DIAMOND (Brandenburg \& Putz, 2012); software used to prepare material for publication: OLEX2 (Dolomanov et al., 2009).

Rubidium peroxide ammonia disolvate

\section{Crystal data}

$\mathrm{Rb}_{2} \mathrm{O}_{2} \cdot 2 \mathrm{NH}_{3}$

$M_{r}=237.01$

Orthorhombic, Pnma

$a=7.3957(7) \AA$

$b=4.0932(6) \AA$

$c=18.1873(17) \AA$

$V=550.57(11) \AA^{3}$

$Z=4$

$F(000)=440$

\section{Data collection}

Agilent SuperNova Dual Source diffractometer with an Eos detector

Mirror monochromator

Detector resolution: 7.9851 pixels $\mathrm{mm}^{-1}$

phi and $\omega$ scans

Absorption correction: analytical

[CrysAlis PRO (Agilent, 2012), based on

expressions derived by Clark \& Reid (1995)]

$T_{\min }=0.064, T_{\max }=0.354$

\section{Refinement}

Refinement on $F^{2}$

Least-squares matrix: full

$R\left[F^{2}>2 \sigma\left(F^{2}\right)\right]=0.050$

$w R\left(F^{2}\right)=0.118$

$S=1.35$

641 reflections

51 parameters

0 restraints
$D_{\mathrm{x}}=2.859 \mathrm{Mg} \mathrm{m}^{-3}$

Mo $K \alpha$ radiation, $\lambda=0.71073 \AA$

Cell parameters from 1711 reflections

$\theta=3.4-28.3^{\circ}$

$\mu=17.66 \mathrm{~mm}^{-1}$

$T=123 \mathrm{~K}$

Block, clear colourless

$0.24 \times 0.09 \times 0.08 \mathrm{~mm}$

2921 measured reflections

641 independent reflections

570 reflections with $I>2 \sigma(I)$

$R_{\text {int }}=0.057$

$\theta_{\text {max }}=26.4^{\circ}, \theta_{\min }=3.6^{\circ}$

$h=-9 \rightarrow 9$

$k=-5 \rightarrow 4$

$l=-22 \rightarrow 22$

Hydrogen site location: difference Fourier map

Only $\mathrm{H}$-atom coordinates refined

$w=1 /\left[\sigma^{2}\left(F_{\mathrm{o}}^{2}\right)+(0.0361 P)^{2}+7.6682 P\right]$

where $P=\left(F_{\mathrm{o}}{ }^{2}+2 F_{\mathrm{c}}{ }^{2}\right) / 3$

$(\Delta / \sigma)_{\max }<0.001$

$\Delta \rho_{\max }=1.12 \mathrm{e} \AA^{-3}$

$\Delta \rho_{\min }=-1.79$ e $\AA^{-3}$ 


\section{Special details}

Geometry. All esds (except the esd in the dihedral angle between two 1.s. planes) are estimated using the full covariance matrix. The cell esds are taken into account individually in the estimation of esds in distances, angles and torsion angles; correlations between esds in cell parameters are only used when they are defined by crystal symmetry. An approximate (isotropic) treatment of cell esds is used for estimating esds involving l.s. planes.

Fractional atomic coordinates and isotropic or equivalent isotropic displacement parameters $\left(\AA^{2}\right)$

\begin{tabular}{llllll}
\hline & $x$ & $y$ & $z$ & $U_{\text {iso }} / U_{\text {eq }}$ & Occ. $(<1)$ \\
\hline $\mathrm{Rb} 1$ & $0.11196(14)$ & 0.2500 & $0.42075(5)$ & $0.0164(4)$ & \\
$\mathrm{Rb} 2$ & $-0.25614(15)$ & 1.2500 & $0.28803(5)$ & $0.0182(4)$ & \\
$\mathrm{O} 1$ & $-0.1266(11)$ & 0.7500 & $0.3825(4)$ & $0.019(2)$ & \\
$\mathrm{O} 2$ & $0.0133(11)$ & 0.7500 & $0.3206(4)$ & $0.022(2)$ & \\
$\mathrm{N} 2$ & $0.6843(16)$ & 0.2500 & $0.4710(6)$ & $0.020(3)$ & 0.5 \\
$\mathrm{~N} 1$ & $0.4060(18)$ & $0.650(4)$ & $0.3327(7)$ & $0.017(4)$ & \\
$\mathrm{H} 1 \mathrm{~A}$ & $0.28(2)$ & 0.7500 & $0.340(8)$ & $0.025^{*}$ & \\
$\mathrm{H} 1 \mathrm{~B}$ & $0.42(2)$ & 0.7500 & $0.285(8)$ & $0.025^{*}$ & \\
$\mathrm{H} 1 \mathrm{C}$ & $0.46(2)$ & 0.7500 & $0.364(8)$ & $0.025^{*}$ & \\
$\mathrm{H} 2 \mathrm{~A}$ & $0.58(2)$ & 0.2500 & $0.472(8)$ & $0.025^{*}$ & \\
$\mathrm{H} 2 \mathrm{~B}$ & $0.739(13)$ & $0.43(3)$ & $0.441(5)$ & $0.025^{*}$ & \\
\hline
\end{tabular}

Atomic displacement parameters $\left(\AA^{2}\right)$

\begin{tabular}{lllllll}
\hline & $U^{11}$ & $U^{22}$ & $U^{33}$ & $U^{12}$ & $U^{13}$ & $U^{23}$ \\
\hline $\mathrm{Rb} 1$ & $0.0107(5)$ & $0.0268(8)$ & $0.0116(5)$ & 0.000 & $-0.0022(4)$ & 0.000 \\
$\mathrm{Rb} 2$ & $0.0088(5)$ & $0.0345(8)$ & $0.0113(5)$ & 0.000 & $0.0003(4)$ & 0.000 \\
$\mathrm{O} 1$ & $0.012(4)$ & $0.027(6)$ & $0.020(4)$ & 0.000 & $0.003(3)$ & 0.000 \\
$\mathrm{O} 2$ & $0.011(4)$ & $0.040(7)$ & $0.014(4)$ & 0.000 & $0.000(3)$ & 0.000 \\
$\mathrm{~N} 2$ & $0.017(5)$ & $0.025(7)$ & $0.018(5)$ & 0.000 & $0.000(4)$ & 0.000 \\
$\mathrm{~N} 1$ & $0.016(6)$ & $0.019(12)$ & $0.016(6)$ & $-0.007(6)$ & $-0.002(5)$ & $0.000(6)$ \\
\hline
\end{tabular}

Geometric parameters $\left(\AA,{ }^{\circ}\right)$

\begin{tabular}{|c|c|c|c|}
\hline $\mathrm{Rb} 1-\mathrm{Rb} 2^{\mathrm{i}}$ & $3.6383(15)$ & $\mathrm{Rb} 2-\mathrm{N} 1^{\mathrm{x}}$ & $3.507(15)$ \\
\hline $\mathrm{Rb} 1-\mathrm{O} 1$ & $2.790(5)$ & $\mathrm{Rb} 2-\mathrm{N} 1^{\mathrm{xiii}}$ & $2.988(14)$ \\
\hline $\mathrm{Rb} 1-\mathrm{O} 1^{\mathrm{ii}}$ & $3.579(8)$ & $\mathrm{Rb} 2-\mathrm{N} 1^{\mathrm{ix}}$ & $2.988(14)$ \\
\hline $\mathrm{Rb} 1-\mathrm{O} 1^{\mathrm{i}}$ & $2.790(6)$ & $\mathrm{O} 1-\mathrm{Rb} 1^{\mathrm{ii}}$ & $3.579(8)$ \\
\hline $\mathrm{Rb} 1-\mathrm{O} 2$ & $2.836(5)$ & $\mathrm{O} 1-\mathrm{Rb} 1^{\text {viii }}$ & $2.790(5)$ \\
\hline $\mathrm{Rb} 1-\mathrm{O} 2^{\mathrm{i}}$ & $2.836(5)$ & $\mathrm{O} 1-\mathrm{Rb} 2^{\mathrm{i}}$ & $2.839(6)$ \\
\hline $\mathrm{Rb} 1-\mathrm{N} 2^{\mathrm{iii}}$ & $3.292(12)$ & $\mathrm{O} 1-\mathrm{O} 2$ & $1.530(11)$ \\
\hline $\mathrm{Rb} 1-\mathrm{N} 2^{\mathrm{iv}}$ & $3.215(8)$ & $\mathrm{O} 2-\mathrm{Rb} 1^{\text {viii }}$ & $2.836(5)$ \\
\hline $\mathrm{Rb} 1-\mathrm{N} 2^{\mathrm{v}}$ & $3.215(8)$ & $\mathrm{O} 2-\mathrm{Rb} 2^{\mathrm{xiv}}$ & $3.316(6)$ \\
\hline $\mathrm{Rb} 1-\mathrm{N} 2^{\mathrm{vi}}$ & $3.215(8)$ & $\mathrm{O} 2-\mathrm{Rb} 2^{\mathrm{xv}}$ & $3.316(6)$ \\
\hline $\mathrm{Rb} 1-\mathrm{N} 1^{\mathrm{vii}}$ & 3.157 (14) & $\mathrm{O} 2-\mathrm{Rb} 2^{\mathrm{i}}$ & $2.917(6)$ \\
\hline $\mathrm{Rb} 1-\mathrm{N} 1$ & 3.157 (14) & $\mathrm{N} 2-\mathrm{Rb} 1^{\mathrm{iv}}$ & $3.215(8)$ \\
\hline $\mathrm{Rb} 2-\mathrm{O} 1$ & $2.839(6)$ & $\mathrm{N} 2-\mathrm{Rb} 1^{\mathrm{xvi}}$ & $3.292(12)$ \\
\hline $\mathrm{Rb} 2-\mathrm{O}^{\mathrm{viii}}$ & $2.839(6)$ & $\mathrm{N} 2-\mathrm{Rb} 1^{\mathrm{xvii}}$ & $3.215(8)$ \\
\hline $\mathrm{Rb} 2-\mathrm{O} 2^{\text {viii }}$ & $2.917(6)$ & $\mathrm{N} 2-\mathrm{Rb} 2^{x v i i i}$ & $3.356(11)$ \\
\hline
\end{tabular}




\begin{tabular}{|c|c|c|c|}
\hline $\mathrm{Rb} 2-\mathrm{O} 2^{\mathrm{ix}}$ & $3.316(6)$ & $\mathrm{N} 1-\mathrm{Rb} 1^{\mathrm{viii}}$ & $3.652(15)$ \\
\hline $\mathrm{Rb} 2-\mathrm{O} 2$ & $2.917(6)$ & $\mathrm{N} 1-\mathrm{Rb} 2^{\mathrm{xiv}}$ & $2.988(14)$ \\
\hline $\mathrm{Rb} 2-\mathrm{O} 2^{\mathrm{x}}$ & $3.316(6)$ & $\mathrm{N} 1-\mathrm{Rb} 2^{\mathrm{xv}}$ & $3.507(15)$ \\
\hline $\mathrm{Rb} 2-\mathrm{N} 2^{\mathrm{xi}}$ & $3.356(11)$ & $\mathrm{N} 1-\mathrm{Rb} 2^{\mathrm{xvi}}$ & $3.598(14)$ \\
\hline $\mathrm{Rb} 2-\mathrm{N} 1^{\mathrm{xi}}$ & $3.095(15)$ & $\mathrm{N} 1-\mathrm{Rb} 2^{\mathrm{xviii}}$ & $3.095(15)$ \\
\hline $\mathrm{Rb} 2-\mathrm{N} 1^{\mathrm{xii}}$ & $3.095(15)$ & $\mathrm{N} 1-\mathrm{N} 1^{\mathrm{xix}}$ & $0.82(3)$ \\
\hline 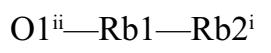 & $133.30(13)$ & $\mathrm{O} 2-\mathrm{Rb} 2-\mathrm{N} 1^{\mathrm{x}}$ & $53.2(3)$ \\
\hline $\mathrm{O} 1^{\mathrm{i}}-\mathrm{Rb} 1-\mathrm{Rb} 2^{\mathrm{i}}$ & $50.32(13)$ & $\mathrm{O} 2-\mathrm{Rb} 2-\mathrm{N} 1^{\mathrm{xi}}$ & $150.4(3)$ \\
\hline $\mathrm{O} 1-\mathrm{Rb} 1-\mathrm{Rb} 2^{\mathrm{i}}$ & $50.32(13)$ & $\mathrm{O} 2-\mathrm{Rb} 2-\mathrm{N} 1^{\mathrm{xii}}$ & $97.3(3)$ \\
\hline 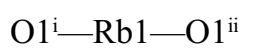 & $105.56(17)$ & $\mathrm{O} 2^{\mathrm{ix}}-\mathrm{Rb} 2-\mathrm{N} 1^{\mathrm{x}}$ & $103.6(3)$ \\
\hline $\mathrm{O} 1-\mathrm{Rb} 1-\mathrm{O} 1^{\mathrm{i}}$ & $94.4(2)$ & $\mathrm{O} 2^{\mathrm{viii}}-\mathrm{Rb} 2-\mathrm{N} 1^{\mathrm{ix}}$ & $59.4(3)$ \\
\hline $\mathrm{O} 1-\mathrm{Rb} 1-\mathrm{O} 1^{\mathrm{ii}}$ & $105.56(17)$ & 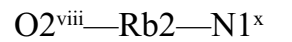 & $112.7(3)$ \\
\hline $\mathrm{O} 1{ }^{\mathrm{i}}-\mathrm{Rb} 1-\mathrm{O} 2$ & $101.92(19)$ & $\mathrm{O} 2^{\mathrm{viii}}-\mathrm{Rb} 2-\mathrm{N} 1^{\mathrm{xi}}$ & $97.3(3)$ \\
\hline $\mathrm{O} 1^{\mathrm{i}}-\mathrm{Rb} 1-\mathrm{O} 2^{\mathrm{i}}$ & $31.5(2)$ & $\mathrm{O} 2^{\mathrm{x}}-\mathrm{Rb} 2-\mathrm{N} 1^{\mathrm{x}}$ & $51.0(3)$ \\
\hline $\mathrm{O} 1-\mathrm{Rb} 1-\mathrm{O} 2$ & $31.5(2)$ & $\mathrm{O} 2-\mathrm{Rb} 2-\mathrm{N} 1^{\mathrm{xiii}}$ & $59.4(3)$ \\
\hline $\mathrm{O} 1-\mathrm{Rb} 1-\mathrm{O} 2^{\mathrm{i}}$ & $101.92(19)$ & 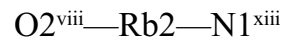 & $105.0(3)$ \\
\hline 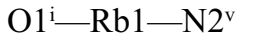 & $156.3(3)$ & $\mathrm{O} 2-\mathrm{Rb} 2-\mathrm{N} 1^{\mathrm{ix}}$ & $105.0(3)$ \\
\hline 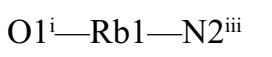 & $57.42(17)$ & $\mathrm{N} 2^{\mathrm{xi}}-\mathrm{Rb} 2-\mathrm{N} 1^{\mathrm{x}}$ & $131.7(3)$ \\
\hline 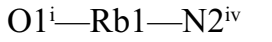 & $156.3(3)$ & $\mathrm{N} 11^{\mathrm{xi}}-\mathrm{Rb} 2-\mathrm{O} 2^{\mathrm{x}}$ & $93.8(3)$ \\
\hline $\mathrm{O} 1-\mathrm{Rb} 1-\mathrm{N} 2^{\mathrm{v}}$ & $89.0(2)$ & $\mathrm{N} 11^{\mathrm{xi}}-\mathrm{Rb} 2-\mathrm{O} 2^{\mathrm{ix}}$ & $54.2(3)$ \\
\hline $\mathrm{O} 1-\mathrm{Rb} 1-\mathrm{N} 2^{\mathrm{iv}}$ & $89.0(2)$ & $\mathrm{N} 1^{\mathrm{ix}}-\mathrm{Rb} 2-\mathrm{O} 2^{\mathrm{ix}}$ & $55.3(3)$ \\
\hline $\mathrm{O} 1-\mathrm{Rb} 1-\mathrm{N} 2^{\mathrm{vi}}$ & $156.3(3)$ & $\mathrm{N} 1^{\mathrm{xii}}-\mathrm{Rb} 2-\mathrm{O} 2^{\mathrm{ix}}$ & $93.8(3)$ \\
\hline $\mathrm{O} 1-\mathrm{Rb} 1-\mathrm{N} 2^{\mathrm{iii}}$ & $57.42(17)$ & $\mathrm{N} 1^{\mathrm{xiii}}-\mathrm{Rb} 2-\mathrm{O} 2^{\mathrm{x}}$ & $55.3(3)$ \\
\hline $\mathrm{O} 1^{\mathrm{i}}-\mathrm{Rb} 1-\mathrm{N} 2^{\mathrm{vi}}$ & 88.98 (19) & $\mathrm{N} 1^{\mathrm{xii}}-\mathrm{Rb} 2-\mathrm{O} 2^{\mathrm{x}}$ & $54.2(3)$ \\
\hline $\mathrm{O} 1-\mathrm{Rb} 1-\mathrm{N} 1$ & $85.9(3)$ & $\mathrm{N} 1^{\mathrm{xiii}}-\mathrm{Rb} 2-\mathrm{O} 2^{\mathrm{ix}}$ & $96.1(3)$ \\
\hline 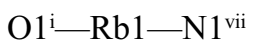 & $85.9(3)$ & $\mathrm{N} 1^{\mathrm{ix}}-\mathrm{Rb} 2-\mathrm{O} 2^{\mathrm{x}}$ & $96.1(3)$ \\
\hline 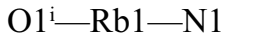 & $133.5(3)$ & $\mathrm{N} 1^{\mathrm{xii}}-\mathrm{Rb} 2-\mathrm{N} 2^{\mathrm{xi}}$ & $68.5(3)$ \\
\hline $\mathrm{O} 1-\mathrm{Rb} 1-\mathrm{N} 1^{\mathrm{vii}}$ & $133.5(3)$ & $\mathrm{N} 1^{\mathrm{xiii}}-\mathrm{Rb} 2-\mathrm{N} 2^{\mathrm{xi}}$ & $141.3(3)$ \\
\hline $\mathrm{O} 2^{\mathrm{i}}-\mathrm{Rb} 1-\mathrm{Rb} 2^{\mathrm{i}}$ & $51.77(13)$ & $\mathrm{N} 1^{\mathrm{xi}}-\mathrm{Rb} 2-\mathrm{N} 2^{\mathrm{xi}}$ & $68.5(3)$ \\
\hline $\mathrm{O} 2-\mathrm{Rb} 1-\mathrm{Rb} 2^{\mathrm{i}}$ & $51.77(13)$ & $\mathrm{N} 1^{\mathrm{ix}}-\mathrm{Rb} 2-\mathrm{N} 2^{\mathrm{xi}}$ & $141.3(3)$ \\
\hline $\mathrm{O} 2-\mathrm{Rb} 1-\mathrm{O} 1^{\mathrm{ii}}$ & $130.55(13)$ & $\mathrm{N} 1^{\mathrm{xii}}-\mathrm{Rb} 2-\mathrm{N} 1^{\mathrm{xi}}$ & $63.8(6)$ \\
\hline 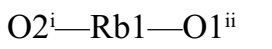 & $130.55(13)$ & $\mathrm{N} 1^{\mathrm{ix}}-\mathrm{Rb} 2-\mathrm{N} 1^{\mathrm{xi}}$ & $103.1(5)$ \\
\hline $\mathrm{O} 2-\mathrm{Rb} 1-\mathrm{O} 2^{\mathrm{i}}$ & $92.4(2)$ & $\mathrm{N} 1^{\mathrm{ix}}-\mathrm{Rb} 2-\mathrm{N} 1^{\mathrm{xii}}$ & $143.7(3)$ \\
\hline $\mathrm{O} 2-\mathrm{Rb} 1-\mathrm{N} 2^{\mathrm{v}}$ & $93.14(17)$ & $\mathrm{N} 1^{\mathrm{xiii}}-\mathrm{Rb} 2-\mathrm{N} 1^{\mathrm{x}}$ & $11.3(4)$ \\
\hline $\mathrm{O} 2^{\mathrm{i}}-\mathrm{Rb} 1-\mathrm{N} 2^{\mathrm{iv}}$ & $166.8(2)$ & $\mathrm{N} 1^{\mathrm{xiii}}-\mathrm{Rb} 2-\mathrm{N} 1^{\mathrm{ix}}$ & $66.4(6)$ \\
\hline $\mathrm{O} 2^{\mathrm{i}}-\mathrm{Rb} 1-\mathrm{N} 2^{\mathrm{vi}}$ & $93.14(17)$ & $\mathrm{N} 1^{\mathrm{ix}}-\mathrm{Rb} 2-\mathrm{N} 1^{\mathrm{x}}$ & $77.7(3)$ \\
\hline $\mathrm{O} 2-\mathrm{Rb} 1-\mathrm{N} 2^{\mathrm{iii}}$ & $86.0(2)$ & $\mathrm{N} 1^{\mathrm{xiii}}-\mathrm{Rb} 2-\mathrm{N} 1^{\mathrm{xii}}$ & $103.1(5)$ \\
\hline $\mathrm{O} 2-\mathrm{Rb} 1-\mathrm{N} 2^{\mathrm{iv}}$ & $93.14(17)$ & 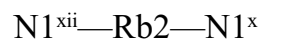 & $94.0(2)$ \\
\hline $\mathrm{O} 2-\mathrm{Rb} 1-\mathrm{N} 2^{\mathrm{vi}}$ & $166.8(2)$ & 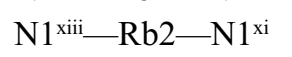 & $143.7(3)$ \\
\hline 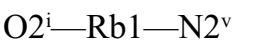 & $166.8(2)$ & $\mathrm{N} 1^{\mathrm{xi}}-\mathrm{Rb} 2-\mathrm{N} 1^{\mathrm{x}}$ & $144.2(4)$ \\
\hline $\mathrm{O} 2^{\mathrm{i}}-\mathrm{Rb} 1-\mathrm{N} 2^{\mathrm{iii}}$ & $86.0(2)$ & $\mathrm{Rb} 1^{\mathrm{viii}}-\mathrm{O} 1-\mathrm{Rb} 1^{\mathrm{ii}}$ & $74.44(17)$ \\
\hline $\mathrm{O} 2-\mathrm{Rb} 1-\mathrm{N} 1$ & $58.5(3)$ & $\mathrm{Rb} 1-\mathrm{O} 1-\mathrm{Rb} 1^{\mathrm{viii}}$ & $94.4(2)$ \\
\hline $\mathrm{O} 2-\mathrm{Rb} 1-\mathrm{N} 1^{\mathrm{vii}}$ & $103.0(3)$ & $\mathrm{Rb} 1-\mathrm{O} 1-\mathrm{Rb} 1^{\mathrm{ii}}$ & $74.44(17)$ \\
\hline $\mathrm{O} 2{ }^{\mathrm{i}}-\mathrm{Rb} 1-\mathrm{N} 1$ & $103.0(3)$ & $\mathrm{Rb} 1-\mathrm{O} 1-\mathrm{Rb} 2^{\mathrm{i}}$ & $80.53(7)$ \\
\hline $\mathrm{O} 2^{\mathrm{i}}-\mathrm{Rb} 1-\mathrm{N} 1^{\mathrm{vii}}$ & $58.5(3)$ & $\mathrm{Rb} 1^{\mathrm{viii}}-\mathrm{O} 1-\mathrm{Rb} 2$ & $80.53(7)$ \\
\hline $\mathrm{N} 2{ }^{\mathrm{ii}}-\mathrm{Rb} 1-\mathrm{Rb} 2^{\mathrm{i}}$ & $57.66(19)$ & $\mathrm{Rb} 1^{\text {viii }-O} 1-\mathrm{Rb} 2^{\mathrm{i}}$ & $153.3(3)$ \\
\hline 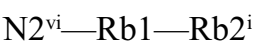 & $139.21(14)$ & $\mathrm{Rb} 1-\mathrm{O} 1-\mathrm{Rb} 2$ & $153.3(3)$ \\
\hline
\end{tabular}




\begin{tabular}{|c|c|}
\hline $\mathrm{N} 2^{\mathrm{iv}}-\mathrm{Rb} 1-\mathrm{Rb} 2^{\mathrm{i}}$ & $139.21(14)$ \\
\hline $\mathrm{N} 2^{\mathrm{v}}-\mathrm{Rb} 1-\mathrm{Rb} 2^{\mathrm{i}}$ & $139.21(14)$ \\
\hline $\mathrm{N} 2^{\mathrm{iv}}-\mathrm{Rb} 1-\mathrm{O} 1^{\mathrm{ii}}$ & $51.21(19)$ \\
\hline $\mathrm{N} 2^{\mathrm{v}}-\mathrm{Rb} 1-\mathrm{O}^{\mathrm{ii}}$ & $51.21(19)$ \\
\hline 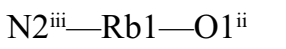 & $75.6(2)$ \\
\hline 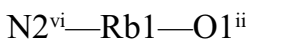 & $51.21(19)$ \\
\hline $\mathrm{N} 2^{\mathrm{v}}-\mathrm{Rb} 1-\mathrm{N} 2^{\mathrm{iii}}$ & $106.3(2)$ \\
\hline 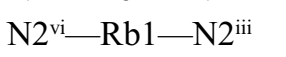 & $106.3(2)$ \\
\hline $\mathrm{N} 2^{\mathrm{iv}}-\mathrm{Rb} 1-\mathrm{N} 2^{\mathrm{iii}}$ & $106.3(2)$ \\
\hline $\mathrm{N} 2^{\mathrm{v}}-\mathrm{Rb} 1-\mathrm{N} 2^{\mathrm{vi}}$ & $79.1(2)$ \\
\hline $\mathrm{N} 2^{\mathrm{v}}-\mathrm{Rb} 1-\mathrm{N} 2^{\mathrm{iv}}$ & $0.0(5)$ \\
\hline $\mathrm{N} 2^{\mathrm{iv}}-\mathrm{Rb} 1-\mathrm{N} 2^{\mathrm{vi}}$ & $79.1(2)$ \\
\hline 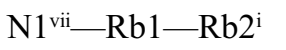 & $100.3(2)$ \\
\hline $\mathrm{N} 1-\mathrm{Rb} 1-\mathrm{Rb} 2^{\mathrm{i}}$ & $100.3(2)$ \\
\hline $\mathrm{N} 1-\mathrm{Rb} 1-\mathrm{O} 1^{\mathrm{ii}}$ & $119.1(3)$ \\
\hline 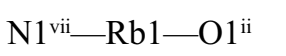 & $119.1(3)$ \\
\hline $\mathrm{N} 1-\mathrm{Rb} 1-\mathrm{N} 2^{\mathrm{v}}$ & $70.0(3)$ \\
\hline $\mathrm{N} 1^{\mathrm{vii}}-\mathrm{Rb} 1-\mathrm{N} 2^{\mathrm{vi}}$ & $70.0(3)$ \\
\hline $\mathrm{N} 1-\mathrm{Rb} 1-\mathrm{N} 2^{\mathrm{iv}}$ & $70.0(3)$ \\
\hline $\mathrm{N} 1^{\mathrm{vii}}-\mathrm{Rb} 1-\mathrm{N} 2^{\mathrm{iv}}$ & $108.5(3)$ \\
\hline $\mathrm{N} 1-\mathrm{Rb} 1-\mathrm{N} 2^{\mathrm{vi}}$ & $108.5(3)$ \\
\hline $\mathrm{N} 1-\mathrm{Rb} 1-\mathrm{N} 2^{\mathrm{iii}}$ & $143.4(3)$ \\
\hline 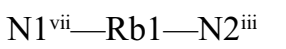 & $143.4(3)$ \\
\hline $\mathrm{N} 1^{\mathrm{vii}}-\mathrm{Rb} 1-\mathrm{N} 2^{\mathrm{v}}$ & $108.5(3)$ \\
\hline $\mathrm{N} 1-\mathrm{Rb} 1-\mathrm{N} 1^{\mathrm{vii}}$ & $62.4(6)$ \\
\hline $\mathrm{O} 1-\mathrm{Rb} 2-\mathrm{O} 1^{\mathrm{viii}}$ & $92.3(2)$ \\
\hline $\mathrm{O} 1^{\text {viii- }-\mathrm{Rb} 2-\mathrm{O} 2^{\mathrm{ix}}}$ & $95.11(15)$ \\
\hline $\mathrm{O} 1-\mathrm{Rb} 2-\mathrm{O} 2^{\mathrm{viii}}$ & $98.78(18)$ \\
\hline $\mathrm{O} 1-\mathrm{Rb} 2-\mathrm{O} 2^{\mathrm{x}}$ & $95.11(15)$ \\
\hline $\mathrm{O} 1^{\mathrm{viii}-\mathrm{Rb} 2-\mathrm{O} 2^{\mathrm{x}}}$ & $168.22(18)$ \\
\hline $\mathrm{O} 1-\mathrm{Rb} 2-\mathrm{O} 2^{\mathrm{ix}}$ & $168.22(18)$ \\
\hline $\mathrm{O} 1^{\text {viii- }} \mathrm{Rb} 2-\mathrm{O} 2^{\text {viii }}$ & $30.8(2)$ \\
\hline $\mathrm{O} 1{ }^{\text {viii- }} \mathrm{Rb} 2-\mathrm{O} 2$ & $98.77(18)$ \\
\hline $\mathrm{O} 1-\mathrm{Rb} 2-\mathrm{O} 2$ & $30.8(2)$ \\
\hline $\mathrm{O} 1-\mathrm{Rb} 2-\mathrm{N} 2^{\mathrm{xi}}$ & $56.23(17)$ \\
\hline 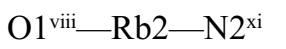 & $56.23(17)$ \\
\hline 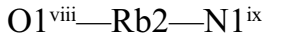 & $85.1(3)$ \\
\hline $\mathrm{O} 1-\mathrm{Rb} 2-\mathrm{N} 1^{1 \mathrm{x}}$ & $134.7(3)$ \\
\hline $\mathrm{O} 1-\mathrm{Rb} 2-\mathrm{N} 1^{\mathrm{x}}$ & $76.0(3)$ \\
\hline $\mathrm{O} 1-\mathrm{Rb} 2-\mathrm{N} 1^{\mathrm{xi}}$ & $119.6(3)$ \\
\hline $\mathrm{O} 1-\mathrm{Rb} 2-\mathrm{N} 1^{\mathrm{xii}}$ & $74.5(3)$ \\
\hline 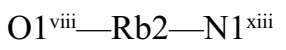 & $134.7(3)$ \\
\hline 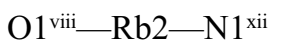 & $119.6(3)$ \\
\hline 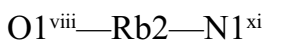 & $74.5(3)$ \\
\hline $\mathrm{O} 1^{\mathrm{vii}}-\mathrm{Rb} 2-\mathrm{N} 1^{\mathrm{x}}$ & $140.3(3)$ \\
\hline $\mathrm{O} 1-\mathrm{Rb} 2-\mathrm{N} 1^{\mathrm{xiii}}$ & $85.1(3)$ \\
\hline $\mathrm{O} 2^{\mathrm{x}}-\mathrm{Rb} 2-\mathrm{O} 2^{\mathrm{ix}}$ & $76.22(17)$ \\
\hline $\mathrm{O} 2-\mathrm{Rb} 2-\mathrm{O} 2^{\mathrm{ix}}$ & $154.83(12)$ \\
\hline
\end{tabular}

\begin{tabular}{|c|c|}
\hline $\mathrm{Rb} 2-\mathrm{O} 1-\mathrm{Rb} 1^{\mathrm{ii}}$ & $127.97(15)$ \\
\hline $\mathrm{Rb} 2^{\mathrm{i}}-\mathrm{O} 1-\mathrm{Rb} 1^{\mathrm{ii}}$ & $127.97(15)$ \\
\hline $\mathrm{Rb} 2-\mathrm{O} 1-\mathrm{Rb} 2$ & $92.3(2)$ \\
\hline $\mathrm{O} 2-\mathrm{O} 1-\mathrm{Rb} 1^{\mathrm{ii}}$ & $135.7(5)$ \\
\hline $\mathrm{O} 2-\mathrm{O} 1-\mathrm{Rb} 1$ & $75.9(3)$ \\
\hline $\mathrm{O} 2-\mathrm{O} 1-\mathrm{Rb} 1^{\mathrm{viii}}$ & $75.9(3)$ \\
\hline $\mathrm{O} 2-\mathrm{O} 1-\mathrm{Rb} 2$ & $77.4(3)$ \\
\hline $\mathrm{O} 2-\mathrm{O} 1-\mathrm{Rb} 2^{\mathrm{i}}$ & $77.4(3)$ \\
\hline $\mathrm{Rb} 1-\mathrm{O} 2-\mathrm{Rb} 1^{\mathrm{viii}}$ & $92.4(2)$ \\
\hline 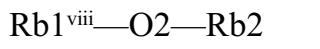 & $78.45(8)$ \\
\hline $\mathrm{Rb} 1-\mathrm{O} 2-\mathrm{Rb} 2$ & $144.3(3)$ \\
\hline $\mathrm{Rb} 1^{\mathrm{viii}}-\mathrm{O} 2-\mathrm{Rb} 2^{\mathrm{xiv}}$ & $134.1(3)$ \\
\hline $\mathrm{Rb} 1-\mathrm{O} 2-\mathrm{Rb} 2^{\mathrm{xiv}}$ & $78.75(10)$ \\
\hline $\mathrm{Rb} 1-\mathrm{O} 2-\mathrm{Rb} 2^{\mathrm{i}}$ & $78.45(8)$ \\
\hline $\mathrm{Rb} 1-\mathrm{O} 2-\mathrm{Rb} 2^{\mathrm{xv}}$ & $134.1(3)$ \\
\hline $\mathrm{Rb} 1^{\text {viii }}-\mathrm{O} 2-\mathrm{Rb} 2^{\mathrm{i}}$ & $144.3(3)$ \\
\hline $\mathrm{Rb} 1^{\mathrm{viii}}-\mathrm{O} 2-\mathrm{Rb} 2^{\mathrm{xv}}$ & $78.75(10)$ \\
\hline $\mathrm{Rb} 2 \mathrm{i}-\mathrm{O} 2-\mathrm{Rb} 2$ & $89.1(2)$ \\
\hline $\mathrm{Rb} 2^{\mathrm{xiv}}-\mathrm{O} 2-\mathrm{Rb} 2^{\mathrm{xv}}$ & $76.22(17)$ \\
\hline $\mathrm{Rb} 2-\mathrm{O} 2-\mathrm{Rb} 2^{\mathrm{xv}}$ & $78.31(10)$ \\
\hline $\mathrm{Rb} 2^{\mathrm{i}}-\mathrm{O} 2-\mathrm{Rb}^{\mathrm{xiv}}$ & $78.32(10)$ \\
\hline $\mathrm{Rb} 2-\mathrm{O} 2-\mathrm{Rb} 2^{\mathrm{xiv}}$ & $131.6(3)$ \\
\hline $\mathrm{Rb} 2^{\mathrm{i}}-\mathrm{O} 2-\mathrm{Rb} 2^{\mathrm{xv}}$ & $131.6(3)$ \\
\hline $\mathrm{O} 1-\mathrm{O} 2-\mathrm{Rb} 1$ & $72.6(3)$ \\
\hline $\mathrm{O} 1-\mathrm{O} 2-\mathrm{Rb} 1^{\mathrm{viii}}$ & $72.6(3)$ \\
\hline $\mathrm{O} 1-\mathrm{O} 2-\mathrm{Rb} 2$ & $71.8(3)$ \\
\hline $\mathrm{O} 1-\mathrm{O} 2-\mathrm{Rb} 2^{\mathrm{xv}}$ & $141.86(9)$ \\
\hline $\mathrm{O} 1-\mathrm{O} 2-\mathrm{Rb} 2^{\mathrm{xiv}}$ & $141.86(9)$ \\
\hline $\mathrm{O} 1-\mathrm{O} 2-\mathrm{Rb} 2^{\mathrm{i}}$ & $71.8(3)$ \\
\hline $\mathrm{Rb} 1^{\mathrm{iv}}-\mathrm{N} 2-\mathrm{Rb} 1^{\mathrm{xvi}}$ & $73.7(2)$ \\
\hline $\mathrm{Rb} 1^{\mathrm{iv}}-\mathrm{N} 2-\mathrm{Rb} 1^{\mathrm{xvii}}$ & $79.1(2)$ \\
\hline $\mathrm{Rb} 1^{\mathrm{xvii}}-\mathrm{N} 2-\mathrm{Rb}^{\mathrm{xvi}}$ & $73.7(2)$ \\
\hline $\mathrm{Rb} 1^{\mathrm{xvi}}-\mathrm{N} 2-\mathrm{Rb} 2^{\mathrm{xviii}}$ & $66.4(2)$ \\
\hline $\mathrm{Rb} 1^{\mathrm{xvii}}-\mathrm{N} 2-\mathrm{Rb} 2^{\mathrm{xviii}}$ & $123.1(3)$ \\
\hline $\mathrm{Rb} 1^{\mathrm{iv}}-\mathrm{N} 2-\mathrm{Rb} 2^{\mathrm{xviii}}$ & $123.1(3)$ \\
\hline $\mathrm{Rb} 1-\mathrm{N} 1-\mathrm{Rb} 1^{\mathrm{viii}}$ & $73.5(3)$ \\
\hline $\mathrm{Rb} 1-\mathrm{N} 1-\mathrm{Rb} 2^{\mathrm{xvi}}$ & $161.2(5)$ \\
\hline $\mathrm{Rb} 1-\mathrm{N} 1-\mathrm{Rb} 2^{\mathrm{xv}}$ & $116.4(4)$ \\
\hline $\mathrm{Rb} 2^{\mathrm{xiv}}-\mathrm{N} 1-\mathrm{Rb} 1$ & $79.2(4)$ \\
\hline $\mathrm{Rb} 2^{\mathrm{xvi}}-\mathrm{N} 1-\mathrm{Rb} 1^{\mathrm{viii}}$ & $93.0(4)$ \\
\hline $\mathrm{Rb} 2^{\mathrm{xv}}-\mathrm{N} 1-\mathrm{Rb} 1^{\mathrm{viii}}$ & $66.4(3)$ \\
\hline $\mathrm{Rb} 2^{\mathrm{xviii}}-\mathrm{N} 1-\mathrm{Rb} 1$ & $114.5(5)$ \\
\hline $\mathrm{Rb} 2^{\mathrm{xiv}}-\mathrm{N} 1-\mathrm{Rb} 1^{\mathrm{viii}}$ & $116.9(4)$ \\
\hline $\mathrm{Rb} 2^{\mathrm{xviii}}-\mathrm{N} 1-\mathrm{Rb} 1^{\text {viii }}$ & $162.1(5)$ \\
\hline $\mathrm{Rb} 2^{\mathrm{xviii}}-\mathrm{N} 1-\mathrm{Rb} 2^{\mathrm{xvi}}$ & $75.0(3)$ \\
\hline $\mathrm{Rb} 2^{\mathrm{xviii}}-\mathrm{N} 1-\mathrm{Rb} 2^{\mathrm{xv}}$ & $118.8(4)$ \\
\hline $\mathrm{Rb} 2^{\mathrm{xv}}-\mathrm{N} 1-\mathrm{Rb} 2^{\mathrm{xvi}}$ & $67.5(3)$ \\
\hline $\mathrm{Rb} 2^{\mathrm{xiv}}-\mathrm{N} 1-\mathrm{Rb} 2^{\mathrm{xv}}$ & $77.7(3)$ \\
\hline
\end{tabular}




\begin{tabular}{|c|c|c|c|}
\hline $\mathrm{O} 2^{\mathrm{viii}}-\mathrm{Rb} 2-\mathrm{O} 2^{\mathrm{ix}}$ & $92.24(11)$ & $\mathrm{Rb} 2^{\mathrm{xiv}}-\mathrm{N} 1-\mathrm{Rb} 2^{\mathrm{xvi}}$ & $119.1(4)$ \\
\hline $\mathrm{O}{ }^{\mathrm{viii}}-\mathrm{Rb} 2-\mathrm{O} 2$ & $89.1(2)$ & $\mathrm{Rb} 2^{\mathrm{xiv}}-\mathrm{N} 1-\mathrm{Rb} 2^{\mathrm{xviii}}$ & 80.9 (4) \\
\hline $\mathrm{O} 2^{\mathrm{viii}}-\mathrm{Rb} 2-\mathrm{O} 2^{\mathrm{x}}$ & $154.83(12)$ & $\mathrm{N} 1^{\mathrm{xix}}-\mathrm{N} 1-\mathrm{Rb} 1^{\mathrm{viii}}$ & $47.7(2)$ \\
\hline $\mathrm{O} 2-\mathrm{Rb} 2-\mathrm{O} 2^{\mathrm{x}}$ & $92.24(11)$ & $\mathrm{N} 1{ }^{\mathrm{xix}}-\mathrm{N} 1-\mathrm{Rb} 1$ & $121.2(3)$ \\
\hline $\mathrm{O} 2^{\mathrm{viii}-\mathrm{Rb} 2-\mathrm{N} 2^{\mathrm{xi}}}$ & $83.6(2)$ & $\mathrm{N} 1^{\mathrm{xix}}-\mathrm{N} 1-\mathrm{Rb} 2^{\mathrm{xv}}$ & $45.5(2)$ \\
\hline $\mathrm{O} 2^{\mathrm{ix}}-\mathrm{Rb} 2-\mathrm{N} 2^{\mathrm{xi}}$ & $121.53(19)$ & $\mathrm{N} 1^{\mathrm{xix}}-\mathrm{N} 1-\mathrm{Rb} 2^{\mathrm{xvi}}$ & $46.9(3)$ \\
\hline $\mathrm{O} 2^{\mathrm{x}}-\mathrm{Rb} 2-\mathrm{N} 2^{\mathrm{xi}}$ & $121.53(19)$ & $\mathrm{N} 1^{\mathrm{xix}}-\mathrm{N} 1-\mathrm{Rb} 2^{\mathrm{xiv}}$ & $123.2(3)$ \\
\hline $\mathrm{O} 2-\mathrm{Rb} 2-\mathrm{N} 2^{\mathrm{xi}}$ & $83.6(2)$ & $\mathrm{N} 1^{\mathrm{xix}}-\mathrm{N} 1-\mathrm{Rb} 2^{\mathrm{xvii}}$ & $121.9(3)$ \\
\hline $\mathrm{O} 2^{\mathrm{vii}}-\mathrm{Rb} 2-\mathrm{N} 1^{\mathrm{xii}}$ & $150.4(3)$ & & \\
\hline
\end{tabular}

Symmetry codes: (i) $x, y-1, z$; (ii) $-x,-y+1,-z+1$; (iii) $x-1, y, z$; (iv) $-x+1,-y+1,-z+1$; (v) $-x+1, y+1 / 2,-z+1$; (vi) $-x+1, y-1 / 2,-z+1$; (vii) $x,-y+1 / 2, z$; (viii) $x, y+1, z$; (ix) $x-1 / 2, y+1,-z+1 / 2$; (x) $x-1 / 2, y,-z+1 / 2$; (xi) $x-1, y+1, z$; (xii) $x-1,-y+3 / 2, z$; (xiii) $x-1 / 2,-y+3 / 2,-z+1 / 2$; (xiv) $x+1 / 2, y-1,-z+1 / 2$; (xv) $x+1 / 2, y,-z+1 / 2$; (xvi) $x+1, y, z$; (xvii) $-x+1,-y,-z+1$; (xviii) $x+1, y-1, z$; (xix) $x,-y+3 / 2, z$.

Hydrogen-bond geometry $\left(\AA,{ }^{\circ}\right)$

\begin{tabular}{lllll}
\hline$D-\mathrm{H} \cdots A$ & $D-\mathrm{H}$ & $\mathrm{H} \cdots A$ & $D \cdots A$ & $D-\mathrm{H} \cdots A$ \\
\hline $\mathrm{N} 1-\mathrm{H} 1 A \cdots \mathrm{O} 2$ & $1.05(14)$ & $1.98(15)$ & $2.941(16)$ & $151(8)$ \\
$\mathrm{N} 1-\mathrm{H} 1 B \cdots \mathrm{O} 2^{\mathrm{xx}}$ & $0.97(13)$ & $2.04(15)$ & $2.926(15)$ & $151(8)$ \\
$\mathrm{N} 1-\mathrm{H} 1 C \cdots \mathrm{O} 1^{\text {vi }}$ & $0.82(14)$ & $3.07(16)$ & $3.597(16)$ & $125(11)$ \\
$\mathrm{N} 2-\mathrm{H} 2 A \cdots \mathrm{N} 2^{\text {iv }}$ & $0.74(16)$ & $3.03(12)$ & $3.57(2)$ & $131(6)$ \\
$\mathrm{N} 2-\mathrm{H} 2 A \cdots \mathrm{N} 2^{\text {vi }}$ & $0.74(16)$ & $3.03(12)$ & $3.57(2)$ & $131(6)$ \\
$\mathrm{N} 2-\mathrm{H} 2 A \cdots \mathrm{N} 2^{\mathrm{v}}$ & $0.74(16)$ & $3.03(12)$ & $3.57(2)$ & $131(6)$ \\
$\mathrm{N} 2-\mathrm{H} 2 B \cdots \mathrm{O} 1^{\text {xiv }}$ & $1.01(11)$ & $1.95(11)$ & $2.955(10)$ & $173(8)$
\end{tabular}

Symmetry codes: (iv) $-x+1,-y+1,-z+1$; (v) $-x+1, y+1 / 2,-z+1$; (vi) $-x+1, y-1 / 2,-z+1$; (xvi) $x+1, y, z$; (xx) $x+1 / 2,-y+3 / 2,-z+1 / 2$. 